\title{
Alcohol per capita Consumption, Patterns of Drinking and Abstention Worldwide after 1995*
}

\begin{tabular}{|c|c|c|c|c|c|c|c|c|}
\hline Country & $\begin{array}{l}\text { Pattern } \\
\text { value }\end{array}$ & $\begin{array}{l}\text { Validity } \\
\text { pattern }\end{array}$ & $\begin{array}{l}\text { Unrecorded } \\
\text { consumption }\end{array}$ & $\begin{array}{l}\text { GNP } \\
\text { per capita }\end{array}$ & $\begin{array}{l}\text { Per capita } \\
\text { consumption }\end{array}$ & $\begin{array}{l}\text { Male } \\
\text { abstainers }\end{array}$ & $\begin{array}{l}\text { Female } \\
\text { abstainers }\end{array}$ & $\begin{array}{l}\text { Validity } \\
\text { abstention }\end{array}$ \\
\hline Albania & 3 & 0 & 3.0 & 803.3 & 5.3 & 12 & 36 & 0 \\
\hline Algeria & 3 & 0 & 0.3 & & 0.5 & 80 & 98 & 0 \\
\hline Argentina & 2 & 1 & 1.0 & $7,970.0$ & 10.5 & 7 & 21 & 1 \\
\hline Armenia & 2 & 0 & 1.9 & 536.7 & 2.9 & 12 & 36 & 0 \\
\hline Australia & 1 & 1 & 0.0 & $20,840.0$ & 9.2 & 7 & 10 & 1 \\
\hline Austria & 1 & 0 & 1.0 & $27,760.0$ & 14.1 & 13 & 33 & 1 \\
\hline Azerbaijan & 3 & 0 & 1.9 & 446.7 & 3.5 & 12 & 36 & 0 \\
\hline Barbados & 2 & 0 & -0.5 & $6,610.0$ & 7.3 & 29 & 70 & 0 \\
\hline Belarus & 4 & 0 & 4.9 & $2,250.0$ & 13.4 & 2 & 4 & 1 \\
\hline Belgium & 1 & 0 & 0.5 & $26,403.3$ & 11.6 & 14 & 27 & 0 \\
\hline Belize & 4 & 0 & 2.0 & $2,700.0$ & 7.5 & 24 & 44 & 0.5 \\
\hline Bolivia & 3 & 0 & 3.0 & 963.3 & 6.2 & 24 & 45 & 1 \\
\hline Bosnia and Herzegovina & 3 & 0 & 3.0 & & 7.7 & 12 & 36 & 0 \\
\hline Botswana & 3 & 0 & 3.0 & $3,250.0$ & 4.9 & 37 & 70 & 0 \\
\hline Brazil & 3 & 1 & 3.0 & $4,586.7$ & 8.9 & 36 & 57 & 1 \\
\hline Bulgaria & 2 & 1 & 3.0 & $1,196.7$ & 14.5 & 12 & 36 & 1 \\
\hline Burkina Faso & & & 3.3 & & 3.9 & & & \\
\hline Burundi & & & 4.7 & & 6.4 & & & \\
\hline Cambodia & 3 & 0 & 0.5 & 273.3 & 0.8 & 74 & 96 & 0 \\
\hline Cameroon & & & 2.6 & & 3.7 & & & \\
\hline Canada & 2 & 1 & 2.0 & $19,506.7$ & 9.7 & 17 & 28 & 1 \\
\hline Central African Republic & & & 1.7 & & 3.3 & & & \\
\hline Chile & 3 & 0 & 2.0 & $4,720.0$ & 8.9 & 31 & 47 & 1 \\
\hline China & 2 & 1 & 1.0 & 690.0 & 4.6 & 16 & 71 & 1 \\
\hline Colombia & 3 & 0 & 2.0 & $2,450.0$ & 8.6 & 31 & 47 & 0.5 \\
\hline Costa Rica & 4 & 0 & 2.0 & $2,696.7$ & 7.5 & 45 & 75 & 1 \\
\hline Croatia & 3 & 0 & 4.5 & $4,526.7$ & 17.6 & 12 & 36 & 0 \\
\hline Cuba & 2 & 0 & 2.0 & & 5.3 & 29 & 70 & 0 \\
\hline Cyprus & 1 & 0 & 1.0 & $12,053.3$ & 9.7 & 1 & 15 & 0 \\
\hline Czech Republic & 2 & 1 & 1.0 & $5,200.0$ & 15.2 & 3 & 8 & 1 \\
\hline Democratic People's Rep. of Korea & & & & & 5.3 & & & \\
\hline Denmark & 1 & 1 & 2.0 & $33,970.0$ & 14.4 & 2 & 4 & 1 \\
\hline Djibouti & & 0.47 & 0.5 & & 0.9 & & & \\
\hline Dominican Republic & 2 & 0 & 1.0 & $1,673.3$ & 5.9 & 29 & 70 & 0 \\
\hline Ecuador & 3 & 0 & 1.0 & $1,520.0$ & 7.9 & 20 & 40 & 0.5 \\
\hline Egypt & 2 & 0 & 0.5 & & 1.0 & 70 & 98 & 0.5 \\
\hline El Salvador & 4 & 0 & 2.0 & $1,780.0$ & 4.7 & 9 & 38 & 0 \\
\hline Eritrea & & & 1.0 & & 2.5 & & & \\
\hline Estonia & 3 & 0 & 5.0 & $3,300.0$ & 7.7 & 5 & 10 & 1 \\
\hline
\end{tabular}

\section{KARGER}

(C) 2001 S. Karger AG, Basel

Fax + 41613061234

E-Mail karger@karger.ch 1022-6877/01/0073-0155\$17.50/0 


\begin{tabular}{|c|c|c|c|c|c|c|c|c|}
\hline Country & $\begin{array}{l}\text { Pattern } \\
\text { value }\end{array}$ & $\begin{array}{l}\text { Validity } \\
\text { pattern }\end{array}$ & $\begin{array}{l}\text { Unrecorded } \\
\text { consumption }\end{array}$ & $\begin{array}{l}\text { GNP } \\
\text { per capita }\end{array}$ & $\begin{array}{l}\text { Per capita } \\
\text { consumption }\end{array}$ & $\begin{array}{l}\text { Male } \\
\text { abstainers }\end{array}$ & $\begin{array}{l}\text { Female } \\
\text { abstainers }\end{array}$ & $\begin{array}{l}\text { Validity } \\
\text { abstention }\end{array}$ \\
\hline Ethiopia & & & 1.0 & & 1.9 & & & \\
\hline Fiji & 3 & 0 & 1.0 & $2,466.7$ & 2.9 & 74 & 98 & 1 \\
\hline Finland & 3 & 1 & 2.0 & $24,033.3$ & 11.8 & 10 & 18 & 1 \\
\hline France & 1 & 1 & 1.0 & $25,073.3$ & 15.7 & 12 & 16 & 0.5 \\
\hline Gabon & & & & & 8.0 & & & \\
\hline Georgia & 2 & 0 & 2.0 & 723.3 & 8.9 & 12 & 36 & 0 \\
\hline Germany & 1 & 1 & 1.0 & $27,760.0$ & 14.5 & 8 & 12 & 1 \\
\hline Ghana & & & 3.6 & & 5.5 & & & \\
\hline Greece & 2 & 1 & 2.0 & $11,683.3$ & 12.6 & 1 & 15 & 1 \\
\hline Guatemala & 4 & 0 & 2.0 & $1,563.3$ & 3.9 & 45 & 62 & 0.5 \\
\hline Guyana & 3 & 0 & 2.0 & 773.3 & 15.5 & 20 & 40 & 0 \\
\hline Haiti & 2 & 0 & 0.0 & 383.3 & 6.3 & 58 & 62 & 0.5 \\
\hline Honduras & 4 & 0 & 2.0 & 700.0 & 4.5 & 9 & 38 & 0 \\
\hline Hungary & 3 & 0 & 4.0 & $4,446.7$ & 18.2 & 7 & 21 & 1 \\
\hline Iceland & 3 & 0 & 1.0 & $27,300.0$ & 6.2 & 9 & 13 & 0 \\
\hline India & 3 & 1 & 1.7 & & 2.0 & 75 & 96 & 1 \\
\hline Indonesia & 3 & 0 & 1.0 & & 1.1 & 74 & 96 & 0 \\
\hline Iraq & 2 & 0 & 0.0 & & 0.6 & 80 & 98 & 0 \\
\hline Ireland & 3 & 1 & 1.0 & $17,836.7$ & 15.5 & 9 & 16 & 1 \\
\hline Israel & 2 & 1 & 1.0 & $16,673.3$ & 2.9 & 21 & 49 & 1 \\
\hline Italy & 1 & 1 & 1.5 & $20,040.0$ & 11.3 & 15 & 30 & 1 \\
\hline Jamaica & 2 & 0 & 1.0 & $2,046.7$ & 5.1 & 29 & 70 & 0 \\
\hline Japan & 1 & 1 & 2.0 & $37,296.7$ & 8.7 & 12 & 23 & 1 \\
\hline Jordan & 2 & 0 & 0.3 & $1,493.3$ & 0.4 & 74 & 98 & 0 \\
\hline Kazakhstan & 4 & 0 & 4.9 & $1,316.7$ & 11.1 & 10 & 27 & 0 \\
\hline Kenya & 3 & 0 & 5.0 & & 7.0 & 45 & 65 & 0 \\
\hline Kyrgyzstan & 3 & 0 & 2.0 & 473.3 & 6.5 & 60 & 80 & 0 \\
\hline Lao People's Democratic Republic & & & & & 5.9 & & & \\
\hline Latvia & 3 & 0 & 7.0 & $2,290.0$ & 18.8 & 15 & 46 & 1 \\
\hline Lebanon & & & & & 4.1 & & & \\
\hline Lesotho & & & 1.5 & & 2.6 & & & \\
\hline Liberia & & & & & 6.0 & & & \\
\hline Lithuania & 3 & 0 & 4.9 & $2,256.7$ & 12.1 & 15 & 46 & 0 \\
\hline Luxembourg & 1 & 0 & -1.0 & $46,443.3$ & 16.6 & 1 & 4 & 0.5 \\
\hline Malaysia & 3 & 0 & 3.4 & $4,186.7$ & 4.4 & 35 & 64 & 1 \\
\hline Mauritius & 3 & 0 & 11.0 & $3,720.0$ & 15.2 & 22 & 53 & 1 \\
\hline Mexico & 4 & 1 & 3.0 & $3,776.7$ & 8.2 & 9 & 38 & 1 \\
\hline Mongolia & 3 & 0 & 2.0 & 383.3 & 4.2 & 20 & 63 & 0 \\
\hline Morocco & & & 1.0 & & 1.7 & & & \\
\hline Myanmar & 2 & 0 & 0.4 & & 0.5 & 45 & 94 & 0 \\
\hline Netherlands & 1 & 1 & 0.5 & $25,590.0$ & 10.4 & 14 & 27 & 1 \\
\hline New Zealand & 1 & 1 & 0.5 & $15,386.7$ & 10.3 & 10 & 14 & 1 \\
\hline Nicaragua & 4 & 0 & 1.0 & 393.3 & 3.7 & 9 & 38 & 0 \\
\hline Nigeria & 2 & 1 & 3.5 & & 4.2 & 45 & 65 & 1 \\
\hline Norway & 3 & 1 & 2.0 & $35,046.7$ & 7.6 & 9 & 13 & 1 \\
\hline Pakistan & 2 & 0 & 0.3 & 503.3 & 0.4 & 90 & 99 & 0 \\
\hline Papua New Guinea & 3 & 1 & 0.5 & $1,016.7$ & 0.9 & 22 & 87 & 1 \\
\hline Paraguay & 3 & 0 & 1.5 & $1,863.3$ & 11.3 & 18 & 38 & 0.5 \\
\hline Peru & 3 & 1 & 1.0 & $2,533.3$ & 5.6 & 17 & 24 & 1 \\
\hline Philippines & 3 & 1 & 3.0 & $1,136.7$ & 6.9 & 10 & 70 & 1 \\
\hline Poland & 3 & 1 & 3.0 & $3,855.0$ & 12.6 & 12 & 26 & 1 \\
\hline Portugal & 1 & 0 & 1.0 & $10,736.7$ & 14.6 & 7 & 24 & 0 \\
\hline Republic of Korea & 3 & 0 & 7.0 & & 14.2 & 12 & 37 & 0.5 \\
\hline Republic of Moldova & 4 & 0 & 12.0 & 476.7 & 30.5 & 9 & 18 & 0 \\
\hline Romania & 3 & 0 & 4.0 & $1,433.3$ & 15.4 & 23 & 53 & 0 \\
\hline Russian Federation & 4 & 1 & 4.9 & $2,400.0$ & 17.5 & 9 & 8 & 1 \\
\hline Rwanda & & & 4.3 & & 5.4 & & & \\
\hline Saudi Arabia & 2 & 0 & 0.6 & & 0.1 & 95 & 99 & 0 \\
\hline Senegal & & & 0.8 & & 1.2 & & & \\
\hline Seychelles & 3 & 1 & 5.2 & & 11.0 & 10 & 45 & 1 \\
\hline Sierra Leone & & & 2.4 & & 2.5 & & & \\
\hline Singapore & 2 & 0 & 1.0 & $31,600.0$ & 3.1 & 74 & 96 & 0 \\
\hline Slovakia & 3 & 1 & 7.0 & $3,590.0$ & 20.2 & 5 & 22 & 1 \\
\hline Slovenia & 2 & 0 & 1.3 & $9,666.7$ & 14.6 & 12 & 36 & 0 \\
\hline
\end{tabular}




\begin{tabular}{|c|c|c|c|c|c|c|c|c|}
\hline Country & $\begin{array}{l}\text { Pattern } \\
\text { value }\end{array}$ & $\begin{array}{l}\text { Validity } \\
\text { pattern }\end{array}$ & $\begin{array}{l}\text { Unrecorded } \\
\text { consumption }\end{array}$ & $\begin{array}{l}\text { GNP } \\
\text { per capita }\end{array}$ & $\begin{array}{l}\text { Per capita } \\
\text { consumption }\end{array}$ & $\begin{array}{l}\text { Male } \\
\text { abstainers }\end{array}$ & $\begin{array}{l}\text { Female } \\
\text { abstainers }\end{array}$ & $\begin{array}{l}\text { Validity } \\
\text { abstention }\end{array}$ \\
\hline South Africa & 3 & 1 & 2.2 & $3,593.3$ & 11.9 & 55 & 83 & 1 \\
\hline Spain & 1 & 1 & 1.0 & $14,343.3$ & 12.7 & 7 & 24 & 1 \\
\hline Sri Lanka & 3 & 0 & 0.5 & 786.7 & 0.7 & 74 & 96 & 1 \\
\hline Sudan & & & 1.0 & & 1.3 & & & \\
\hline Suriname & 3 & 0 & 0.0 & $1,346.7$ & 6.1 & 30 & 55 & 0.5 \\
\hline Swaziland & & & 4.1 & & 7.0 & & & \\
\hline Sweden & 3 & 1 & 2.0 & $25,903.3$ & 9.1 & 7 & 16 & 1 \\
\hline Switzerland & 1 & 1 & 0.5 & $42,303.3$ & 12.4 & 11 & 27 & 1 \\
\hline Syrian Arab Republic & & & 0.4 & & 0.5 & & & \\
\hline Tajikistan & 3 & 0 & 4.0 & 273.3 & 5.7 & 60 & 80 & 0 \\
\hline TFYR Macedonia & 3 & 0 & 2.9 & $1,553.3$ & 9.7 & 12 & 36 & 0 \\
\hline Thailand & 3 & 1 & 2.0 & $2,583.3$ & 11.2 & 31 & 72 & 1 \\
\hline Trinidad and Tobago & 2 & 1 & 0.0 & $4,203.3$ & 3.3 & 29 & 70 & 1 \\
\hline Tunisia & 2 & 0 & 0.5 & & 1.6 & 70 & 95 & 0 \\
\hline Turkey & 3 & 0 & 2.7 & $3,080.0$ & 4.3 & 35 & 55 & 0 \\
\hline Turkmenistan & 3 & 0 & 1.0 & 656.7 & 2.4 & 35 & 55 & 0 \\
\hline Uganda & 3 & 0 & 10.7 & 306.7 & 11.4 & 45 & 67 & 0.5 \\
\hline Ukraine & 3 & 1 & 8.0 & $1,033.0$ & 8.3 & 12 & 36 & 0 \\
\hline United Arab Emirates & & & 1.0 & & 4.3 & & & \\
\hline United Kingdom & 2 & 1 & 2.0 & $20,613.3$ & 12.0 & 8 & 14 & 1 \\
\hline United Republic of Tanzania & & & 2.0 & & 7.4 & & & \\
\hline United States of America & 2 & 1 & 1.0 & & 9.8 & 28 & 43 & 1 \\
\hline Uruguay & 3 & 0 & 2.0 & $6,016.7$ & 8.3 & 7 & 21 & 0 \\
\hline Uzbekistan & 3 & 0 & 1.9 & 733.3 & 4.5 & 60 & 80 & 0 \\
\hline Venezuela & 3 & 0 & 2.0 & & 10.2 & 30 & 55 & 0.5 \\
\hline Vietnam & & & 1.0 & & 2.4 & & & \\
\hline Zambia & 4 & 1 & 1.0 & & 4.1 & 35 & 70 & 1 \\
\hline Zimbabwe & 4 & 0 & 9.0 & 676.7 & 13.0 & 7 & 36 & 0.5 \\
\hline
\end{tabular}

Country: TFYR Macedonia means The Former Yugoslav Republic of Macedonia.

Pattern value: 1 denotes the best pattern value and 4 denotes the most detrimental, based on the optimal scaling of ratings of key experts on several pattern variables (see Rehm, Monteiro, Room et al., this issue for detailed explanations). The following algorithm was used: in case WHO received a full questionnaire from the country based on survey data, these data were used, even if other experts outside the country doubt the values, unless they can cite a data source. Pattern values are assumed to be relatively stable and were derived for the 1990s.

Validity of pattern value: 0 = Imputed by regional proximity, cultural similarity and/or based on data from the Global Status Report on Alcohol; 1 = questionnaire available with sufficient non-missing values to allow optimal scaling.

Unrecorded consumption: Estimated volume of unrecorded consumption in litres pure alcohol per capita for population older than 15 for the years after 1995 (based on Global Status Report on Alcohol mainly).

GNP per capita: Gross national product per capita after 1995 (average of years in countries with values for more than one year UN data).

Per capita consumption: Average per capita consumption in litres pure alcohol after 1995 including estimates of unrecorded consumption (average of available years).

Male abstainers: Proportion of adult males who had been abstaining (last year before the survey).

Female abstainers: Proportion of adult females who had been abstaining (last year before the survey).

Validity abstention: $0=$ Imputed based on regional and cultural similarities; $0.5=$ based on survey but only for subsections of the country or not differentiating for sex; 1 = survey data, differentiated for sex. 